Boll. Accademia Gioenia di

Scienze Naturali - Catania
BOLLAG Vol. 52, N. 382 (2019)

Difesa ecosostenibile delle colture agricole pp. DECA5 - DECA9

ISSN 0393-7143

\title{
Aspetti normativi e legislazione fitosanitaria a sostegno della difesa eco-sostenibile delle colture ${ }^{\dagger}$
}

\author{
Anna Di Natale $[1]^{*}$ \\ [1] Servizio Fitosanitario Regionale - U.O. S4.04 - Osservatorio per le Malattie delle Piante di Acireale
}

\begin{abstract}
Riassunto
La normativa europea e nazionale in materia fitosanitaria si è inizialmente interessata alla tutela della salute dei consumatori, regolamentando soprattutto la produzione e la commercializzazione dei prodotti fitosanitari e considerando i loro residui negli alimenti. Successivamente, la legislazione europea ha considerato anche l'impatto delle sostanze chimiche contenute nei formulati commerciali nei confronti dell'operatore e dell'ambiente. Inoltre, con l'approvazione della Direttiva 128 del 2009, che "istituisce un quadro per l'azione comunitaria ai fini dell'utilizzo sostenibile dei pesticidi”, per la prima volta ne viene regolamentato l'impiego, stabilendo principi comuni per un loro utilizzo razionale e sicuro. Con questa importante direttiva, si definiscono dei Piani di Azione Nazionale (PAN) per promuovere l'utilizzo di prodotti fitosanitari maggiormente sostenibili e fornire indicazioni per ridurre il loro impatto in ambito agricolo, extra agricolo e in aree naturali protette. In tale contesto, vengono illustrati e discussi i criteri di applicazione e il quadro normativo di riferimento più aggiornato.
\end{abstract}

Parole chiave: sostenibilità dei prodotti fitosanitari, normativa fitosanitaria

\section{Regulatory aspects and phytosanitary legislation to support the eco-sustainable crop protection}

\section{Summary}

The European and Italian phytosanitary legislation has been initially focused on the consumer protection, regulating the production and trade security of pesticides and considering the risk of their residues in the food. Afterward, European regulation has also considered the impact of chemicals on workers and environment. With the approval of Directive 128/2009, which "establishes a framework for community action for the sustainable use of pesticides", for the first time its use is regulated, establishing common principles for their rational and safe use. With this important directive, National Action Plans are defined to promote the use of the most sustainable pesticides and provide indications to reduce their impact in agricultural, extra-agricultural and natural areas. In this context, the application criteria and the most updated regulatory reference framework are described and discussed.

Key words: sustainability of pesticides, phytosanitary regulation and legislation

\footnotetext{
${ }^{\dagger}$ Nota presentata nell'Adunanza pubblica del 15 novembre 2018

*e-mail: anna.dinatale@ regione.sicilia.it
} 


\section{Premessa}

La normativa relativa all'immissione in commercio e all'uso dei prodotti fitosanitari nel tempo si è evoluta tenendo conto sia delle esigenze di sicurezza dei consumatori e dell'ambiente, oltreché dello sviluppo delle coscienze ambientaliste. Il progresso delle tecniche agronomiche e della ricerca scientifica ha permesso di passare da una lotta chimica a calendario (preventiva, anche in assenza di parassiti) alla gestione delle avversità con mezzi alternativi (agronomici, fisici, biologici, ecc.) contenendo le perdite di produzione quanti-qualitative entro limiti tollerabili. Si è così arrivati al controllo delle malattie parassitarie e dei fitofagi ammettendo l'uso di sostanze chimiche solo quando tali mezzi non consentono un efficace contenimento dei parassiti: l'insieme delle strategie utilizzabili prende il nome di difesa integrata.

\section{La normativa}

In Italia, la normativa di riferimento sull'immissione in commercio dei prodotti fitosanitari fino agli anni '90 è stata rappresentata dal Decreto del Presidente della Repubblica 1255 del 3 agosto 1968. Dal 1991 la legislazione è stata regimata in modo armonizzato in tutti gli Stati membri della Comunità europea, secondo un processo comunitario di valutazione e di approvazione delle sostanze attive (restando ai singoli Stati l'autorizzazione dei formulati commerciali). La Direttiva 414 del 1991 è stata così approvata con gli obiettivi di consentire la libera circolazione delle merci all'interno della Comunità, evitare vantaggi competitivi di alcuni Stati rispetto ad altri, ma soprattutto garantire livelli di salute e sicurezza per l'uomo e l'ambiente uguali nell'intero territorio comunitario. La Direttiva 414 del 1991 in Italia è stata recepita con il Decreto legislativo 194 del 1995, a cui sono seguite diverse integrazioni. In particolare, grazie al Decreto n. 290 del 2001 sono state riviste le procedure per l'acquisizione dell'abilitazione alla vendita di tutti i prodotti fitosanitari per gli esercenti e dell'autorizzazione all'acquisto e all'uso dei prodotti fitosanitari più pericolosi da parte degli operatori.

Tuttavia la Direttiva 414 del 1991 non ha raggiunto alcuni degli obiettivi che si era prefissata. Sulla base delle indicazioni del VI programma comunitario di azione in materia ambientale 2002 - 2012, che ha perseguito l'obiettivo della riduzione dei pericoli e dei rischi derivanti dall'impiego dei prodotti fitosanitari secondo specifiche strategie tematiche, nel 2009 sono state approvate nuove norme specificamente finalizzate all'incremento del livello di tutela dell'operatore, del consumatore e dell'ambiente. Queste norme, insieme al Regolamento sui Residui Massimi Ammessi (Regolamento 396 del 2005), costituiscono il cosiddetto pacchetto fitosanitari: la loro prerogativa consiste nella reciproca integrazione con prescrizioni armonizzate fra loro.

In particolare: il Regolamento $\mathbf{1 1 0 7}$ stabilisce criteri ancora più rigorosi per la registrazione e l'immissione in commercio dei prodotti fitosanitari; la Direttiva 127 detta nuovi criteri per l'immissione in commercio delle macchine irroratrici, introducendo requisiti essenziali di protezione ambientale da applicare alla progettazione e costruzione delle nuove macchine per la distribuzione dei prodotti fitosanitari; il Regolamento 1185 introduce lo strumento delle indagini statistiche per la valutazione dell'efficacia delle misure adottate e infine la Direttiva 128 valuta gli aspetti applicativi dei prodotti fitosanitari.

Fino al 2009 la normativa europea e nazionale si è occupata di disciplinare la produzione e la commercializzazione dei prodotti fitosanitari, considerando anche i loro residui negli alimenti. Ma con l'approvazione della Direttiva 128 del 2009, che "istituisce un quadro per l'azione comunitaria ai fini dell'utilizzo sostenibile dei pesticidi", per la prima volta ne viene regolamentato anche l'impiego, stabilendo principi comuni al fine di renderlo più razionale e sicuro. Con questa direttiva, tra l'altro, si richiede agli Stati membri di definire dei Piani di Azione Nazionale per promuovere pratiche di utilizzo dei prodotti fitosanitari maggiormente sostenibili e fornire indicazioni per ridurre l'impatto di questi mezzi di difesa nelle aree agricole, nelle aree extra agricole (aree verdi urbane, strade, ferrovie, ecc.) e nelle aree naturali protette. 
L'Italia ha recepito la direttiva sui pesticidi con il Decreto legislativo 14 agosto 2012, n. 150. Con questa norma, tra l'altro, si identificano le figure professionali che per operare necessitano di una adeguata preparazione, a seguito della quale ottengono una specifica attestazione:

a) l'utilizzatore professionale, che è la persona che utilizza i prodotti fitosanitari nel corso di un'attività professionale;

b) il distributore: che è persona fisica o giuridica in possesso del certificato di abilitazione alla vendita;

c) il consulente: persona in possesso del certificato di abilitazione alle prestazioni di consulenza in materia di difesa integrata.

Si introduce quindi il concetto di difesa integrata che deve essere adottata da tutti gli utilizzatori di prodotti fitosanitari (difesa integrata obbligatoria). In Italia, molte aziende agricole aderiscono a programmi agroambientali seguendo specifiche norme tecniche (disciplinari di produzione) che prevedono una maggiore riduzione dell'uso dei mezzi chimici e forniscono indicazioni sulle possibili alternative (difesa integrata volontaria). Chi non rispetta le indicazioni riportate nel decreto può incorrere in sanzioni amministrative, nonchè sospensione da sei mesi ad un anno fino alla revoca del certificato di abilitazione.

Il Piano di Azione Nazionale (PAN) in Italia è stato pubblicato con il Decreto legislativo 22 gennaio 2014. Questo documento definisce gli obiettivi quantitativi, le misure e i tempi richiesti per un utilizzo sostenibile dei prodotti fitosanitari; come previsto dal Decreto legislativo 150 del 2012, il PAN, che ha durata quinquennale, contiene azioni concrete e misurabili che devono essere facilmente accessibili e comprese dagli operatori e dai cittadini e che sono così sintetizzate:

A.1 - Formazione e prescrizioni per gli utilizzatori, i distributori e i consulenti

A.2 - Informazione e sensibilizzazione

A.3 - Controlli delle attrezzature per l'applicazione dei Prodotti Fitosanitari

A.4 - Irrorazione aerea

A.5 - Misure specifiche per la tutela dell'ambiente acquatico e dell' acqua potabile e per la riduzione dell'uso di prodotti fitosanitari in aree specifiche (rete ferroviaria e stradale, aree frequentate dalla popolazione, aree naturali protette)

A.6 - Manipolazione e stoccaggio dei prodotti fitosanitari e trattamento dei relativi imballaggi e delle rimanenze

A.7 - Difesa fitosanitaria a basso apporto di prodotti fitosanitari (strategie fitosanitarie sostenibili)

Nel corso del 2019 è previsto l'aggiornamento del PAN, che terrà particolarmente conto, tra l'altro, della tutela della biodiversità.

Con il PAN si riconosce l'importanza strategica dell'agricoltura biologica per la salvaguardia degli ecosistemi e la valorizzazione delle biodiversità. Questo metodo di produzione, che è stato introdotto in Europa con l'applicazione del Regolamento 2092 del 1991, e finora disciplinato dal Regolamento 834 del 2007, tende a valorizzare le risorse naturali e prevede l'uso di un limitato numero di prodotti fitosanitari costituiti da sostanze attive di origine naturale. È già stato pubblicato il nuovo aggiornamento della normativa (il Regolamento 848 del 2018), che a partire dal 1 gennaio 2021 introdurrà nuove disposizioni.

A completamento del quadro normativo relativo agli agrofarmaci ed al loro utilizzo, è stato infine pubblicato, con il Decreto 22 gennaio 2018 n. 33, il Regolamento che definisce misure e requisiti dei prodotti fitosanitari destinati agli utilizzatori non professionali, con lo scopo di prevenire i rischi dall'uso scorretto di tali prodotti ai quali possono essere esposti uomo, ambiente e organismi non bersaglio. I prodotti ad uso non professionale vengono classificati in:

- PFnPO: prodotti per la difesa fitosanitaria di piante ornamentali, di libera vendita

- PFnPE: prodotti per la difesa fitosanitaria di piante edibili che devono essere venduti presso una rivendita autorizzata. 


\section{Conclusioni}

Partendo dagli anni novanta l'evoluzione della normativa comunitaria e nazionale ha portato a grandi cambiamenti sull'immissione in commercio e sull'utilizzo dei prodotti fitosanitari. Si è assistito a un processo di revisione caratterizzato dalla progressiva riduzione o limitazione nell'utilizzo di sostanze attive e prodotti riconosciuti pericolosi per l'uomo e per l'ambiente, e nel contempo si è arrivati ad una diminuzione costante delle irregolarità dovute alla presenza di residui di sostanze potenzialmente tossiche nei prodotti agroalimentari. Nell'elaborazione del quadro normativo descritto, i legislatori, a livello comunitario e nazionale, hanno seguito specifiche direttive strategiche, che hanno sempre tenuto conto dei tre criteri che identificano lo sviluppo agricolo sostenibile: redditività economica, accettazione sociale e compatibilità ambientale.

Per il raggiungimento degli obiettivi prestabiliti dalla normativa rimane fondamentale la ricerca scientifica al fine di individuare nuove tecniche necessarie allo sviluppo di strategie di difesa integrata che garantiscono la produttività in agricoltura e nello stesso tempo assicurano la tutela dell' ambiente e della biodiversità.

\section{Legislazione di riferimento}

Di seguito si riporta una sintesi della normativa europea e nazionale che regolamenta l'immissione in commercio e l'utilizzo dei prodotti fitosanitari e dei loro residui negli alimenti: si ricorda che si tratta di una materia in continua evoluzione che richiede un attento e costante aggiornamento.

Decreto del Presidente della Repubblica del 3 agosto 1968, n. 1255 - Regolamento concernente la disciplina della produzione, del commercio e della vendita di fitofarmaci e dei presidi delle derrate alimentari immagazzinate (pubblicato su GURI n. 327 del 27 dicembre 1968, Supplemento Ordinario $n$. 3270)

Direttiva 79/117/CE del Consiglio del 21 dicembre 1978 - Relativa al divieto di immettere in commercio e impiegare prodotti fitosanitari contenenti determinate sostanze attive (pubblicata su GU delle Comunità Europee L 33 del 08 febbraio 1979)

Regolamento (CEE) N. 2092/91 del Consiglio del 24 giugno 1991 - Relativo al metodo di produzione biologico di prodotti agricoli e alla indicazione di tale metodo sui prodotti agricoli e sulle derrate alimentari (pubblicato su GU delle Comunità Europee L 198 del 22.7.1991)

Direttiva del Consiglio del 15 luglio 1991, n. 414 - Relativa all'immissione in commercio dei prodotti fitosanitari (pubblicata su GU della Comunità Europea L 230 del 19 agosto 1991)

Decreto legislativo del 17 marzo 1995, n. 194 - Attuazione della Direttiva 91/414/CEE in materia di immissione in commercio di prodotti fitosanitari (pubblicata su GURI n. 122 del 27 maggio 1995, Supplemento Ordinario n. 60)

Circolare del Ministero della Sanità 15 aprile 1999, n. 7 - Immissione in commercio di prodotti fitosanitari destinati al trattamento delle piante ornamentali e dei fiori da balcone, da appartamento e da giardino domestico, già disciplinati come presidi medico-chirurgici (pubblicato sulla GURI del 13 maggio 1999, n. 110)

Decreto del Presidente della Repubblica del 23 aprile 2001, n. 290 - Regolamento di semplificazione dei procedimenti di autorizzazione alla produzione, alla immissione in commercio e alla vendita dei prodotti fitosanitari e relativi coadiuvanti (pubblicato su GURI n. 165 del 18 luglio 2001, Supplemento Ordinario n. 190)

Regolamento (CE) 396/2005 del Parlamento Europeo e del Consiglio del 23 febbraio 2005 Concernente i livelli massimi di residui di antiparassitari nei o sui prodotti alimentari e mangimi di origine vegetale e animale e che modifica la direttiva 91/414 del Consiglio (pubblicato su $G U$ dell'Unione Europea L 70 del 16 marzo 2005)

Regolamento (CE) n. 834/2007 del Consiglio del 28 giugno 2007 - Relativo alla produzione biologica e all'etichettatura dei prodotti biologici e che abroga il regolamento (CEE) n. 2092/91 (pubblicato su GU dell'Unione Europea L 189 del 20 luglio 2007) 
Regolamento (CE) 889/2008 della Commissione del 5 settembre 2008 - Relativo alla produzione biologica e all'etichettatura dei prodotti biologici, per quanto riguarda la produzione biologica, l'etichettatura e i controlli (pubblicato su GU dell'Unione Europea L 250 del 18 settembre 2008) Regolamento (CE) 1107/2009 del Parlamento Europeo e del Consiglio del 21 ottobre 2009 Relativo all'immissione sul mercato dei prodotti fitosanitari e che abroga le direttive del Consiglio 79/117/CEE e 91/414/CEE (pubblicato su GU dell'Unione Europea L 309/1 del 24 novembre 2009)

Direttiva 2009/128/CE del Parlamento Europeo e del Consiglio del 21 ottobre 2009 - Che istituisce un quadro per l'azione comunitaria ai fini dell'utilizzo sostenibile dei pesticidi (pubblicata su GU dell'Unione Europea L 309 del 24 novembre 2009)

Direttiva 2009/127/CE del Parlamento Europeo e del Consiglio del 21 ottobre 2009 - Che modifica la direttiva 2006/42/CE relativa alle macchine per l'applicazione di pesticidi (pubblicata su GU dell'Unione Europea L 310 del 25 novembre 2009)

Regolamento (CE) n. 1185/2009 del Parlamento Europeo e del Consiglio del 25 novembre 2009 - Relativo alle statistiche sui pesticidi (pubblicato su GU dell'Unione Europea L 324 del 10 dicembre 2009)

Decreto del Presidente della Repubblica 28 febbraio 2012, n. 55 - Regolamento recante modifiche al decreto del Presidente della Repubblica 23 aprile 2001, n. 290, per la semplificazione dei procedimenti di autorizzazione alla produzione, alla immissione in commercio e alla vendita di prodotti fitosanitari e relativi coadiuvanti (pubblicato su GURI n. 109 del 15 maggio 2012)

Decreto Legislativo 14 agosto 2012, n. 150 - Attuazione della direttiva 2009/128/CE che istituisce un quadro per l'azione comunitaria ai fini dell'utilizzo sostenibile dei pesticidi (pubblicato su GURI n. 202 del 30 agosto 2012)

Decreto Legislativo 22 gennaio 2014 - Adozione del Piano di azione nazionale per l'uso sostenibile dei prodotti fitosanitari, ai sensi dell'articolo 6 del decreto legislativo 14 agosto 2012, n. 150 recante: «Attuazione della direttiva 2009/128/CE che istituisce un quadro per l'azione comunitaria ai fini dell'utilizzo sostenibile dei pesticidi» (pubblicato su GURI n. 35 del 12 febbraio 2014)

Decreto Legislativo 17 aprile 2014, n. 69 - Disciplina sanzionatoria per la violazione delle disposizioni del Regolamento (CE) 1107/2009 relativo all'immissione sul mercato dei prodotti fitosanitari e che abroga le direttive 79/117/CEE e 91/414/CEE, nonché del regolamento (CE9 n. $1107 / 2009$ per quanto concerne le prescrizioni in materia di etichettatura ei prodotti fitosanitari (pubblicato sulla GURI del 6 maggio 2014, n. 103)

Regolamento (UE) 848/2018 del Parlamento Europeo e del Consiglio del 30 maggio 2018 Relativo alla produzione biologica e all'etichettatura dei prodotti biologici e che abroga il regolamento (CE) n. 834/2007 del Consiglio (pubblicato su GU dell'Unione Europea L 150/1 del 14 giugno 2018)

Decreto 22 gennaio 2018, n. 33 - Regolamento sulle misure e sui requisiti dei prodotti fitosanitari per un uso sicuro da parte degli utilizzatori non professionali (pubblicato sulla GURI del 6 aprile $2018, n .88)$ 